\title{
Extracellular Vesicles Derived from Hypoxic Colorectal Cancer Cells Confer Metastatic Phenotype to Non-metastatic Cancer Cells
}

\author{
EDGARS ENDZELIN̦Š ${ }^{1}$, ARTŪRS ĀBOLS ${ }^{1}$, ARTŪRS BUŠS ${ }^{1}$, ELĪNA ZANDBERGA ${ }^{1}$, \\ MARI PALVIAINEN ${ }^{2,3}$, PIA SILJANDER ${ }^{2,3,4}$ and AIJA LINE $\bar{E}^{1}$ \\ ${ }^{1}$ Latvian Biomedical Research and Study Centre, Riga, Latvia; \\ ${ }^{2}$ EV-core, University of Helsinki, Helsinki, Finland; \\ ${ }^{3}$ Drug Research Program, Faculty of Pharmacy, Division of Pharmaceutical Biosciences, \\ University of Helsinki, Helsinki, Finland; \\ ${ }^{4}$ Molecular and Integrative Biosciences Research Programme, University of Helsinki, Helsinki, Finland
}

\begin{abstract}
Background/Aim: Tumor-secreted extracellular vesicles (EVs) play an important role as mediators of intercellular communication. Hypoxia is a common feature of solid tumors frequently associated with an aggressive clinical behavior. This study aimed to gain a deeper understanding into the functions of EVs in intercellular communication between primary and metastatic cancer cells under hypoxic conditions. Materials and Methods: EVs were isolated from two isogenic colorectal cancer (CRC) cell lines SW480 and SW620 cultured under normoxic and hypoxic conditions. Their uptake and effects in SW480 and SW620 cells were studied using EV uptake, proliferation, spheroidformation, wound healing and invasion assays. Results: Our data showed that hypoxia enhanced the release of EVs from CRC cells in a Hypoxia Induced Factor (HIF)-1-dependent manner. Hypoxic EVs were taken up by CRC cells more efficiently than normoxic EVs. Hypoxic EVs stimulated motility, invasiveness and stemness of primary tumourderived SW480 cells, whereas they had a little effect on metastasis-derived SW620 cells. Conclusion: Hypoxic colorectal cancer-derived EVs confer aggressiveness and invasiveness to hypoxia-naïve cancer cells.
\end{abstract}

Hypoxia is a common property of the majority of solid cancers, including colorectal cancer (CRC). Accumulating evidence shows that up to $50-60 \%$ of locally advanced solid tumors have hypoxic tissue areas $\left(\mathrm{pO}_{2} \leq 2.5 \mathrm{mmHg}\right)$

Correspondence to: Aija Linē, Ph.D., Latvian Biomedical Research and Study Centre, Ratsupites Str 1, k-1, LV-1067, Riga, Latvia. Tel: +37167808208, e-mail: aija@biomed.lu.lv

Key Words: Extracellular vesicles, colorectal cancer, hypoxia.
(1). Hypoxic areas temporally arise in the tumor tissues due to rapid proliferation of cancer cells and abnormal vasculature (2). Majority of cells exposed to acute hypoxia halt their proliferation and/or undergo apoptotic or necrotic cell death (1). However, under sustained hypoxia a fraction of cancer cells survive and acquire capabilities that enable them to overcome nutrient and oxygen deprivation and/or escape from the hostile environment (1). Clinically, hypoxia is associated with metastasis, drug resistance and poor prognosis (3-5). Intracellular hypoxia signaling is mediated by hypoxia-inducible factors (HIFs) transcription factors that exist as heterodimers formed of an $\alpha$-subunit (HIF- $1 \alpha$, HIF- $2 \alpha$ or HIF $3 \alpha$ ) and $\beta$-subunit (HIF-1 $\beta$ ). The stability of HIF $\alpha$ protein is controlled by oxygen level, while HIF- $1 \beta$ is constitutively expressed. Under normoxic conditions, HIF $\alpha$ interacts with VHL protein, which activates the ubiquitin ligase system leading to the degradation of HIF $\alpha$ by the $26 \mathrm{~S}$ proteasome, while under hypoxic conditions, it is stabilized and forms dimers with HIF-1 $\beta$ (2, 4, 6). Upon dimerization, HIF is translocated to the nucleus, where it controls the expression of genes involved in invasion, metastasis, angiogenesis, stemness, dormancy and metabolic reprogramming $(2,5)$. However, given that the hypoxic areas are typically located in the central parts of the tumors, it is not clear if the cells exposed to hypoxia can exit the tumor and subsequently spread to secondary sites by themselves or whether they contribute to the metastatic progression mainly via signaling to the cells located at the invasive front.

Extracellular vesicles (EVs) have recently emerged as important mediators of the communication between cells. EVs are a heterogeneous population of lipid bilayer-bound vesicles released into the extracellular space by normal and cancer cells. Three main types of EVs are exosomes, 
microvesicles and apoptotic bodies that differ in their biogenesis, and probably also in their molecular content, membrane composition and specific functions $(7,8)$. The molecular content of EVs, at least partially, reflects that of the parent cells. EVs transfer a variety of lipids, proteins, mRNAs, non-coding and structural RNAs and even DNA fragments to recipient cells, thereby influencing their physiological functions $(7,9)$. EVs produced by normal cells are required for the maintenance of homeostasis and the regulation of various physiological functions (7), whereas cancer-derived EVs have been shown to promote tumor growth and metastasis by influencing properties of cancer cells, immune cells, and stromal cells in the tumor microenvironment and at the pre-metastatic niches (10-12). CRC-derived EVs have been shown to stimulate proliferation, motility, colony-forming efficiency and chemoresistance of cancer cells (13-15) and elicit various tumor-promoting effects in endothelial cells (16), mesenchymal stromal cells (17) and immune cells (18).

The current study investigated the effects of EVs released by two isogenic CRC cell lines SW480 and SW620, that were cultured under normoxic and hypoxic conditions on hypoxia-naïve CRC cells. These cell lines were originally established from the primary tumor and the lymph node metastasis obtained from the same patient and therefore represent an in vitro model for studying the molecular mechanisms of CRC metastasis (19). Results showed that hypoxic EVs were more efficiently taken-up by hypoxianaïve SW480 and SW620 cells than normoxic EVs, and hypoxic EVs promoted motility, invasion and spheroidforming capacity of primary tumor-derived SW480 cells.

\section{Materials and Methods}

Cell culture and exposure to hypoxia. The human colorectal adenocarcinoma cell lines SW480 and SW620 were purchased from the American Type Culture Collection (ATCC, USA). The cells were maintained in DMEM/F12 medium (Lonza, Basel, Switzerland, \#BE12-79F) supplemented with 10\% Foetal Bovine Serum (SigmaAldrich, St. Louis, MO, USA, \#F7524) and 1× Antibiotic-Antimycotic (Thermo Fisher Scientific, Waltham, MA, USA, \#15240-062) as adherent monolayer. The cell cultures were monitored for mycoplasma infection using PCR Mycoplasma Test Kit I/C (PromoCell GmbH, Heidelberg, Germany, \#PK-CA91-1096). For EV isolation, the cells were seeded at a density $1 \times 106$ cells per $\mathrm{ml}$ of serum-free DMEM/F12 medium supplemented with $10 \mathrm{ng} / \mathrm{ml}$ bFGF (Santa Cruz Biotechnology, Dallas, TX, USA, \#sc-4573), 20 ng/ml EGF (R\&D Systems, Minneapolis, MN, USA, \#236-EG-200), $0.5 \mu \mathrm{g} / \mathrm{ml}$ hydrocortisone (Sigma-Aldrich, \#H0135), 1× Antibiotic-Antimycotic (Thermo Fisher Scientific, \#15240-062) and 1×B-27 (Thermo Fisher Scientific, \#17504001), and grown as multicellular spheroids for $48 \mathrm{~h}$. To establish hypoxic conditions, the cells were cultured at $1 \% \mathrm{O}_{2}, 94 \%$ $\mathrm{N}_{2}$ and $5 \% \mathrm{CO}_{2}$ in a humidified multi-gas incubator (SANYO Electric Co., Osaka, Japan, \#MCO-5M). To test the effects of HIF-1 inhibition on the EV release, cells were treated with $300 \mu \mathrm{M}$ chetomin (SigmaAldrich, \#C9623) for $48 \mathrm{~h}$.
Immunofluorescence. After $48 \mathrm{~h}$ exposure to hypoxia, multicellular spheroids were washed with PBS, centrifuged onto poly-L-lysinecoated slides (Tharmac GmbH, Waldsolms, Germany, \#JC312) using a Shandon cytospin at $1000 \mathrm{~g}$ for $5 \mathrm{~min}$ and fixed in cold methanol/acetone $(1: 1)$ for $20 \mathrm{~min}$. The slides were blocked with $2 \%$ BSA in PBS for $30 \mathrm{~min}$ and incubated with primary antibodies - rabbit anti- CAIX (Abcam, Cambridge, UK, \#ab15086) (dilution 1:50) or mouse monoclonal anti-HIF1 $\alpha$ (R\&D Systems, \#MAB1935) (dilution 1:50) overnight at $+4^{\circ} \mathrm{C}$. After washing, the slides were incubated with secondary antibodies - goat anti-rabbit IgG conjugated with fluorescein (FITC) (Jackson ImmunoResearch, Cambridgeshire, UK, \#11-095-006) or goat anti-mouse IgG conjugated with cyanine (Cy3) (Jackson ImmunoResearch, \#115165-0) (dilution 1:200) for $1 \mathrm{~h}$ at room temperature. The slides were mounted with ProLong Gold Antifade Mountant with DAPI (Thermo Fisher Scientific, \#P36935) and images were acquired using Leica DM3000 microscope (Leica Microsystems GmbH, Wetzlar, Germany).

EV isolation. EVs were isolated from the conditioned cell culture media using size exclusion chromatography (SEC) as described before (20). Briefly, culture media were centrifuged at $300 \mathrm{~g}$ for $10 \mathrm{~min}$ to remove cells and at $3,000 \times \mathrm{g}$ for $30 \mathrm{~min}$ at $+4^{\circ} \mathrm{C}$ to remove cell debris, then filtered through $0.2 \mu \mathrm{m}$ filters (Sarstedt, Nümbrecht, Germany, \#83.1826.001) and concentrated up to $1 \mathrm{ml}$ using $100 \mathrm{kDa}$ centrifugal filters (Merck Millipore, MA, USA, UFC910024). The media were loaded on CL6B sepharose-filled $10 \mathrm{ml}$ SEC columns and 12 sequential $0.5 \mathrm{ml}$ fractions were collected. Each SEC fraction was measured by Zetasizer Nano ZS (Malvern, UK) and the fractions containing particles from 30 to $200 \mathrm{~nm}$ were pooled, concentrated up to $100 \mu \mathrm{l}$ using 3-kDa filters (Merck, Millipore, UFC500324), aliquoted to avoid repeated freeze/thaw cycles and stored at $-20^{\circ} \mathrm{C}$. EV protein concentration was measured by Pierce BCA Protein Assay Kit (Thermo Fisher Scientific, \#23227).

Western blot. Cells and EVs were lysed in RIPA buffer (50 mM Tris, $\mathrm{pH} 8.0,0.6 \mathrm{M} \mathrm{NaCl}, 4 \%$ Triton $\mathrm{X}-100,2 \%$ sodium deoxycholate, $0.1 \%$ SDS) and the protein concentration was determined using a BCA Protein Assay kit (Pierce, Thermo Fisher Scientific). Ten micrograms of total protein were loaded per lane, separated by $10 \%$ SDS-PAGE and electroblotted to nitrocellulose membranes. The membranes were blocked with $10 \%(\mathrm{w} / \mathrm{v})$ fat-free milk and then incubated with the following primary antibodies: HIF1 $\alpha(1: 2000)$ (Abcam, \#ab51608), CAIX (1:1,000) (Abcam, \#ab15086), ALIX (1:1000) (Santa Cruz, \#sc-53540), TSG101 (1:1,000) (Abcam, \#ab125011), CD9 (1:500) (Santa Cruz, sc-13118), Calnexin $(1: 1,000)$ (Abcam, \#ab22595) and $\beta$-actin $(1: 4,000)$ (Abcam, \#ab8224). The membranes were washed in TBS, $0.05 \%$ Tween and incubated with peroxidase - conjugated rabbit anti-mouse secondary antibody $(1: 2,000)$ (Santa Cruz, \#sc-516102) or mouse anti-rabbit secondary antibody $(1: 2,000)$ (Santa Cruz, \#sc-2357) and processed with ECL Select Western Blotting Detection Reagents (GE Healthcare, USA) according to manufacturer's instructions.

Nanoparticle tracking analysis. Isolated EVs were evaluated by nanoparticle tracking analysis (NTA) using Nanosight instrument LM14 (Malvern, UK) equipped with violet (404 nm, $70 \mathrm{~mW}$ ) laser and SCMOS camera. The samples were diluted in filtered PBS to achieve particle concentration in range from $1 \times 10^{8}$ to $1 \times 10^{9}$ 
particles/ml and three $60 \mathrm{sec}$ videos were recorded using camera level 14. The data were analyzed using NTA software v3.0 with the detection threshold 10 and screen gain at 5 .

Labelling of EVs and EV uptake assay. Isolated EVs (10 $\mu \mathrm{g}$ protein that corresponds to $1.7 \times 10^{9} \pm 3.2 \times 10^{8}$ particles according to NTA data) were stained with $0.5 \mu \mathrm{l}$ PKH67 dye (Sigma- Aldrich, \#MINI67) in $250 \mu \mathrm{l}$ Diluent $\mathrm{C}$ buffer for $5 \mathrm{~min}$ at room temperature. The staining reaction was stopped by adding $500 \mu \mathrm{l}$ of $1 \%$ BSA in PBS. Labelled EVs were washed six times with $500 \mu$ PBS by centrifuging at 14,000 g for 6 min using $300 \mathrm{kDa}$ centrifugal filter units (Pall Corporation, \#OD300C33). As a negative control for the uptake experiments, the PKH67 dye without EVs was processed and used in parallel.

SW480 and SW620 cells were seeded in DMEM/F12 complete medium at a density of $1 \times 10^{5}$ cells per well in 12 -well plates and grown as a monolayer for $24 \mathrm{~h}$ at hypoxic or normoxic conditions. PKH67-labelled EVs (10 $\mu$ g protein) were added to the cells and incubated for $24 \mathrm{~h}$ at $37^{\circ} \mathrm{C}$. Subsequently, cells were washed with PBS, harvested using $1 \times$ trypsin (Thermo Fisher Scientific, \#15090046) and analyzed with the BD FACSAria II instrument (BD Biosciences, BD FACSAria II). The negative control and auto fluorescence percentage was subtracted from the results. The assay was performed in duplicate at two independent times.

For confocal microscopy analysis, $2 \times 10^{5} \mathrm{SW} 480$ cells were seeded on glass coverslips. After $24 \mathrm{~h}$, the growth medium was changed and PKH67-labelled SW480-derived EVs (10 $\mu$ g protein) were added to the cell culture and incubated for another $24 \mathrm{~h}$. Subsequently, cells were washed three times with the growth media, fixed with $4 \%$ buffered FA solution for $10 \mathrm{~min}$ and mounted on glass slides in ProLong ${ }^{\mathrm{TM}}$ Gold Antifade Mountant with DAPI (Thermo Fisher Scientific, P36935). Confocal fluorescence imaging was done on Leica TCS SP8 confocal laser scanning microscopy platform.

Proliferation assay. SW480 and SW620 cells were seeded at a density of $5 \times 10^{3}$ cells per well in DMEM/F12 medium in 96-well plate. After $24 \mathrm{~h}$ of incubation, $10 \mu \mathrm{g}$ of normoxic and hypoxic SW480 and SW620-derived EVs were added to the cells and incubated for $24 \mathrm{~h}$ at $37^{\circ} \mathrm{C}, 5 \% \mathrm{CO}_{2}$. Next, $10 \mu \mathrm{l}$ of the CCK8 solution (Sigma-Aldrich, \#96992) were added to each well and incubated for $4 \mathrm{~h}$ at $37^{\circ} \mathrm{C}, 5 \% \mathrm{CO}_{2}$. The absorbance was measured at $450 \mathrm{~nm}$ using a microplate reader (Perkin Elmer, Victor) and the values were background subtracted. The assay was performed in triplicates at two independent times.

Scratch wound healing assay. SW480 and SW620 cells were seeded in DMEM/F12 complete medium at a density of $2 \times 10^{5}$ cells per well in 24-well plates and grown until they reached $\sim 70-80 \%$ confluence. Scratches were created by scraping the monolayer with a sterile $200 \mu \mathrm{l}$ pipette tip, the wells were washed with PBS to remove detached cells and refiled with fresh medium. EVs $(20 \mu \mathrm{g}$ protein) were added to the cells and photos of the wounds were taken at $0 \mathrm{~h}$ and $24 \mathrm{~h}$. Migration distance was measured using ImageJ software (Leica, Fiji). The assay was performed in triplicates at two independent times.

Cell invasion assay. SW480 and SW620 cell invasion through basement membrane extract (BME) was tested using Cultrex BME Cell Invasion Assay (R\&D Systems, \#3455-096-K) according to the manufacturer's protocol. Briefly, a filter insert (pore size of $8 \mu \mathrm{m}$ ) was coated with $50 \mu \mathrm{l}$ of $0.5 \times \mathrm{BME}$ overnight at $37^{\circ} \mathrm{C}$ in $\mathrm{CO}_{2}$ incubator. On the next day, $5 \times 10^{4}$ cells in $50 \mu \mathrm{l}$ of serum-free medium containing EVs $(10 \mu \mathrm{g}$ protein) were added to the upper chambers, the bottom chambers were filled with $150 \mu \mathrm{l}$ of medium containing $10 \% \mathrm{FBS}$, and incubated for $48 \mathrm{~h}$ at $37^{\circ} \mathrm{C}$. The cells that migrated through the BME layer were dissociated from the membrane, incubated with Calcein AM for $1 \mathrm{~h}$ and the absorbance was measured at $450 \mathrm{~nm}$ using a microplate reader (Perkin Elmer, Victor). The assay was performed in six replicates at two independent times.

Spheroid formation assay. SW480 and SW620 cells were seeded in DMEM/F12 complete medium at a density of $1 \times 105$ cells per well in 6-well plates, grown for $24 \mathrm{~h}$ and then incubated with EVs $(10 \mu \mathrm{g}$ protein) for $24 \mathrm{~h}$. Subsequently, the cells were plated at a density of 250 cells per well in 96-well ultra-low attachment plates (Corning, NY, USA) in serum-free DMEM/F12 medium supplemented with $1 \times B 27$ (Invitrogen, 17504001), $20 \mathrm{ng} / \mathrm{ml}$ EGF (R\&D Systems, USA), $10 \mathrm{ng} / \mathrm{ml} \mathrm{bFGF} \mathrm{(R \& D} \mathrm{Systems,} \mathrm{USA)} \mathrm{and} \mathrm{1 \%} \mathrm{methylcellulose}$ (Sigma-Aldrich, USA) and cultured under normoxic or hypoxic conditions. After 7 days in culture, the spheroids containing at least 50 cells were counted under an inverted microscope (Motic, \#AE31E). Clonal origin of SW480 spheroids was verified by coculturing of DiD (Thermo Fisher Scientific, \#V22887) and DiO (Thermo Fisher Scientific, \#V22886) labelled cells at a density of 250 cells per well. The assay was performed in six replicates at two independent times.

Statistical analysis. All statistical analyses were performed using GraphPad Prism software version 7 (GraphPad Software, Inc, USA). The data are represented in graphs as means \pm SD of two independent experiments each performed in replicates. Statistical significance was determined by the nonparametric Mann-Whitney $U$-test and the differences were considered significant at $p<0.05$.

\section{Results}

Characteristics of normoxic and hypoxic EVs. EVs were isolated by a combination of centrifugation, filtration and SEC from the conditioned medium of human isogenic CRC cell lines SW480 and SW620, which had been cultured as multicellular spheroids under either normoxic or hypoxic conditions. The hypoxic conditioning of the cells was verified by immunofluorescence (Figure 1A) and western blot analysis (Figure 1B) both of which showed a strong induction of two hypoxia markers - HIF $1 \alpha$ and CAIX in the hypoxia-exposed, but not in the normoxia grown spheroids. Isolated EVs were characterized by western blot analysis, transmission electron microscopy (TEM) and NTA. Western blot analysis showed that SW480 and SW620-derived EVs were positive for classic EV markers ALIX and TSG101 (Figure 1B). Compared to the cells, EVs were enriched in CD9, but negative for the endoplasmic reticulum protein calnexin, thus showing that the EV preparations did not contain detectable amounts of intracellular components. Interestingly, hypoxic EVs were strongly enriched in carbonic anhydrase IX (CAIX) - a hypoxia-inducible 


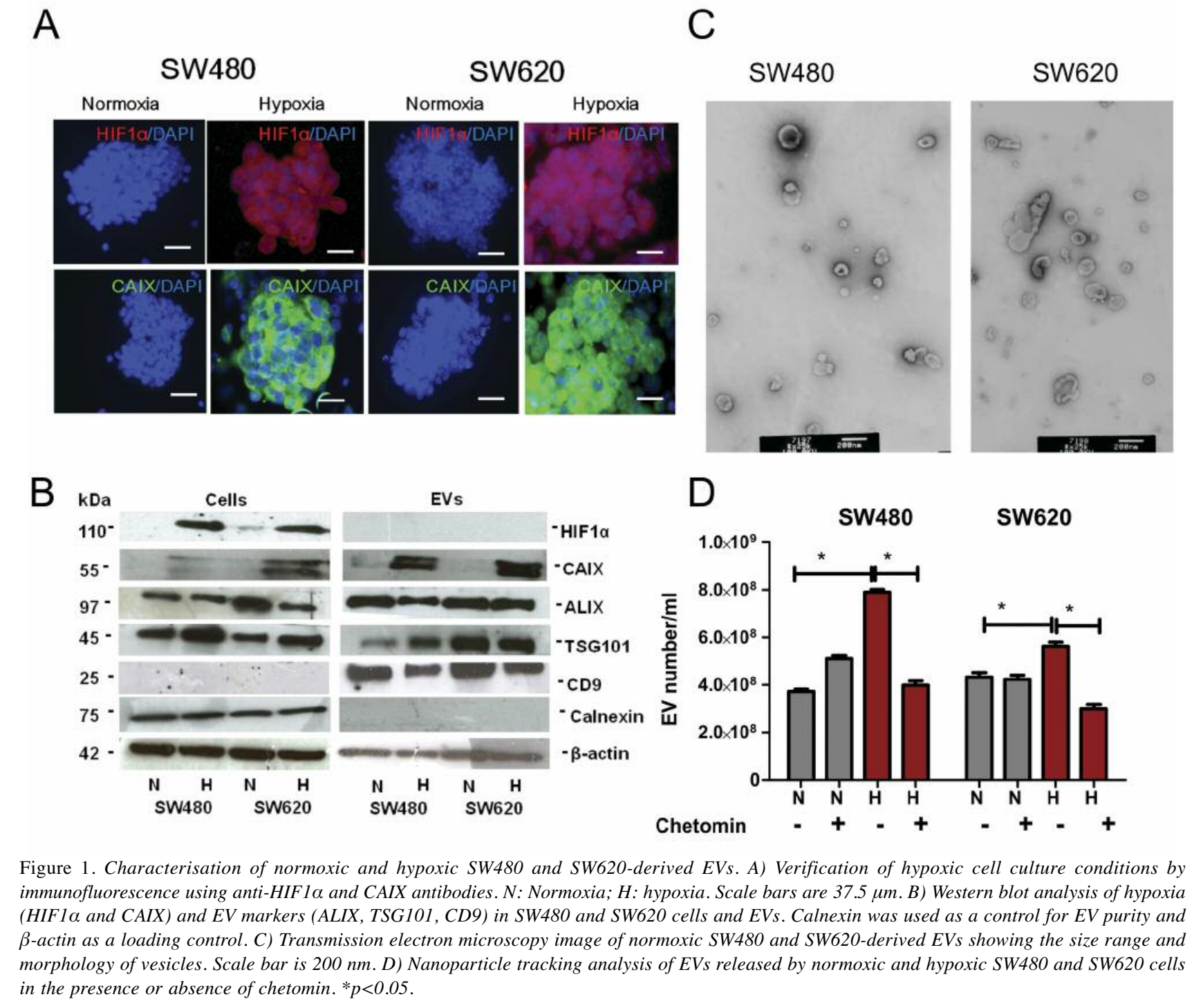

C

enzyme with an extracellular catalytic domain that is overexpressed in various cancers and plays a crucial role in the regulation of intracellular and extracellular pH (21). TEM images showed that the majority of vesicles were 40 to $200 \mathrm{~nm}$ in diameter (Figure 1C). NTA revealed a significant increase in the number of EVs released under hypoxic conditions by SW480 and SW620 cells (2.12-fold, $p=0.05$ and 1.3 -fold, $p=0.05$, respectively) (Figure 1D), while the size distributions were not significantly different between the conditions. To test whether the increased production of EVs depended on HIF signaling, cells were grown in the presence of chetomin, an inhibitor of HIF-1 that disrupts the binding of HIF- $1 \alpha$ and HIF- $2 \alpha$ to the coactivator p300 (22). NTA showed that the treatment with chetomin abrogated the hypoxia-induced increase in the EV production in both cell lines, thus linking the HIF signaling with the increased release of EVs (Figure 1D).

EV interactions with hypoxia-naïve CRC cells. To investigate the interaction/uptake of normoxic and hypoxic EVs by hypoxia-naïve cancer cells, SW480 and SW620 cells were incubated with PKH67-labelled EVs for $24 \mathrm{~h}$ and analyzed by flow cytometry. The results showed that hypoxic EVs interacted with SW480 and SW620 cells more efficiently than normoxic EVs (Figure 2A). The uptake assay was also performed at hypoxic conditions, however no significant differences between the conditions were observed. This suggested that the EV surface molecule composition was altered under hypoxic conditions in a way that facilitated their interaction and/or uptake by cancer cells. Furthermore, 
A
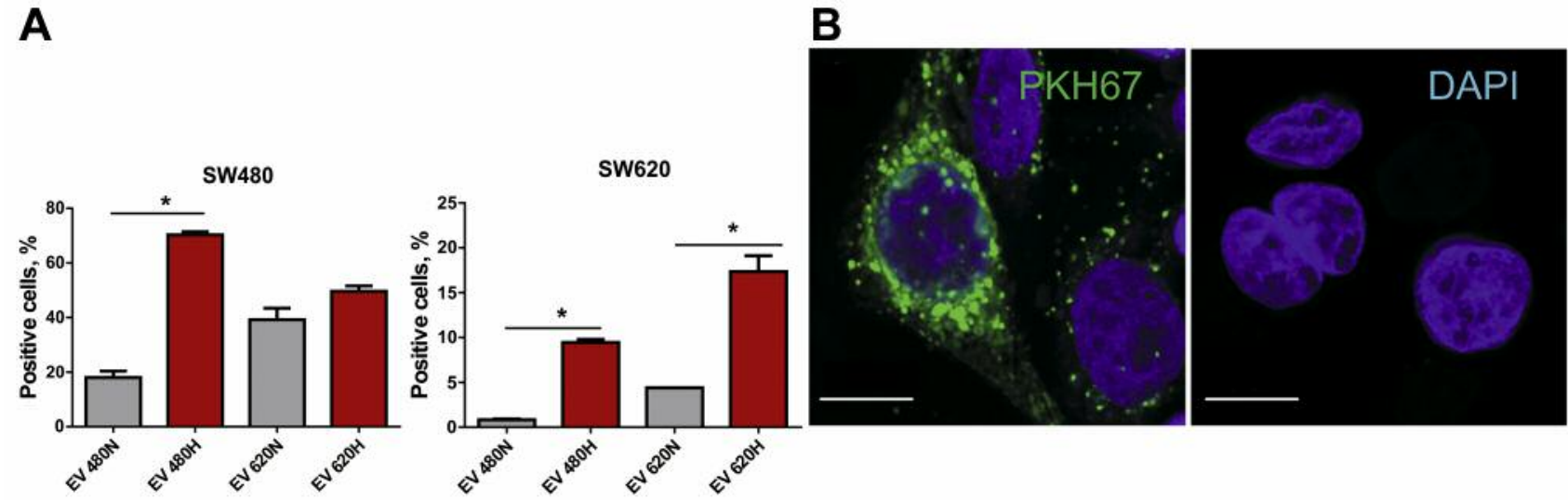

Figure 2. Uptake of normoxic and hypoxic EVs by hypoxia-nä̈ve CRC cells. A) Percentage of PKH67-positive SW480 and SW620 cells after incubation with PKH67-labelled EVs for 24 h determined by flow cytometry analysis. B) EV uptake by SW480 cells visualized by confocal microscopy. Left panel-PKH67-labelled EVs; right-negative control. Scale bar is $10 \mu m .{ }^{*} p<0.05$.

A
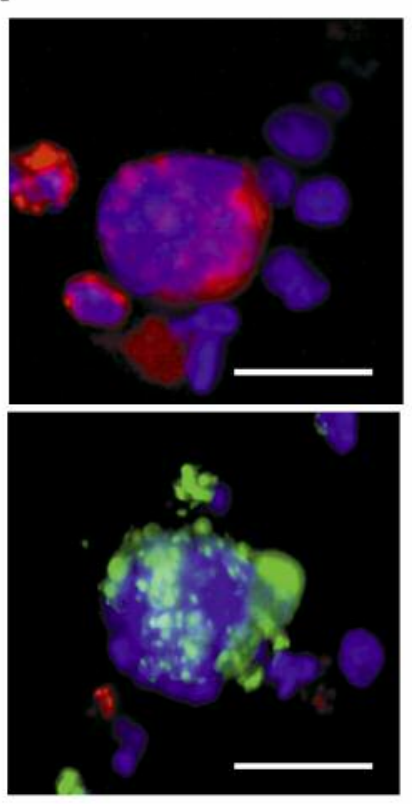

B
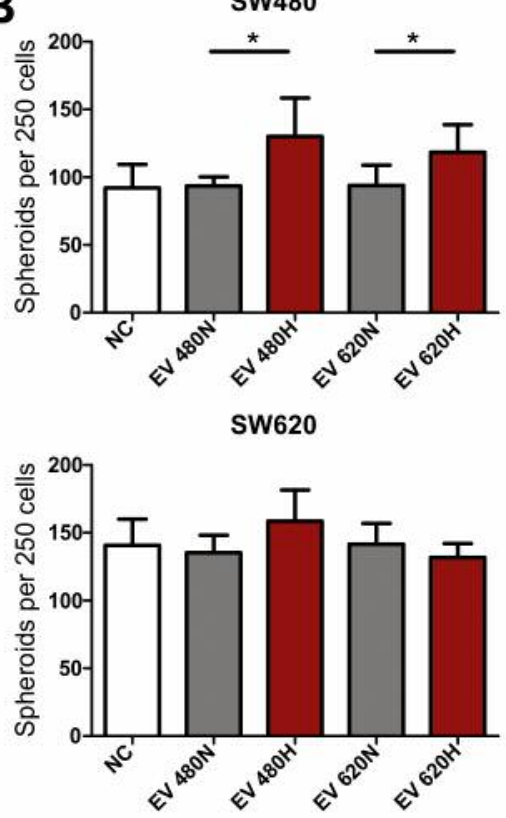

Figure 3. Effects of EVs on spheroid-forming ability of CRC cells. A) The clonal origin of 3D multicellular spheroids was verified by co-culturing of DiD and DiO-labelled SW480 cells. Mostly single-labelled spheroids were observed, when the cells were plated in methylcellulose-containing serum-free medium at a density of 250 cells/well. Scale bar is $37.5 \mu \mathrm{m}$. B) The number of multicellular spheroids formed by 250 SW480 and SW620 cells after 7 days of culturing in methylcellulose-containing serum-free-defined medium. ${ }^{*} p<0.05$.

the interaction/uptake of EVs (independently of hypoxia status) by SW480 was substantially higher than that by SW620 cells, thus suggesting that cancer-derived EVs interact with primary tumor cells more efficiently than with metastatic cells.
To further investigate the mode of interaction of EVs with hypoxia-naïve SW480 cells, PKH67-lebelled hypoxic SW480-derived EVs were incubated with SW480 cells for $24 \mathrm{~h}$ and analyzed by confocal microscopy. PKH67-lebelled EVs were internalized and located in the cytoplasm of 
A

SW480 + 1xPBS

Oh

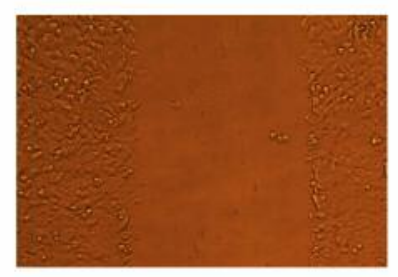

$24 h$

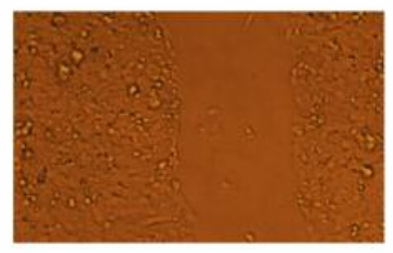

SW480

B

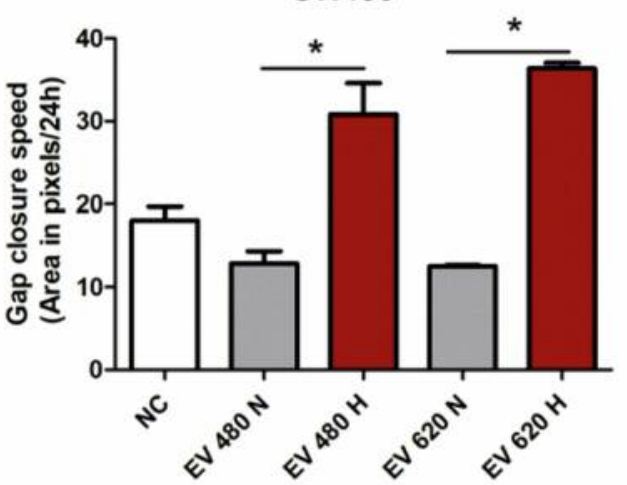

C

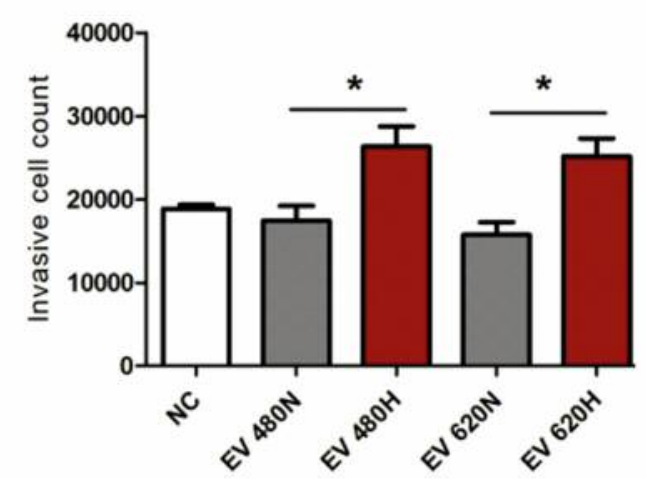

SW480 + EV62OH
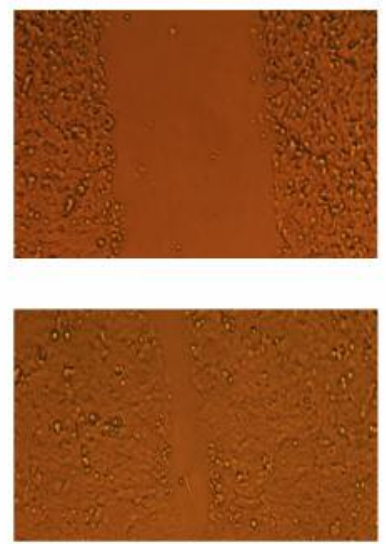

SW620

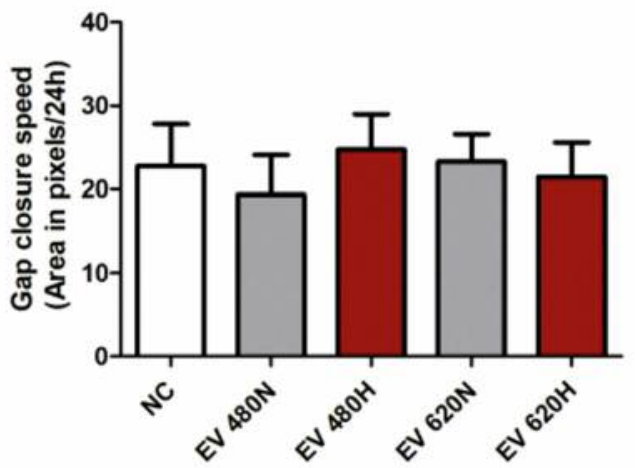

SW620

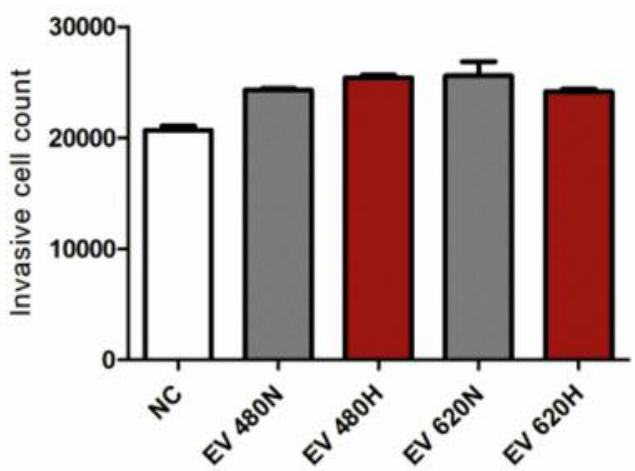

Figure 4. Effects of normoxic and hypoxic EVs on the motility and invasiveness of CRC cells. A) Representative images of scratch wound healing assay of SW480 cells treated with hypoxic and normoxic SW620-derived EVs. B) Graphs showing gap closure after 24 h. C) BME cell invasion assay showing the number of cells that migrated through BME coated membranes to the bottom chamber quantified using Calcein AM. *p<0.05.

SW480 cells. To rule out the transfer of unincorporated PKH67 dye, cells were incubated with a negative control PKH67 dye that was subjected to the EV labelling protocol (Figure 2B).
Effects of EVs on proliferation and spheroid forming ability of CRC cells. To assess the effects of EVs on the growth properties of CRC cells, proliferation and spheroid-forming assays were performed. The proliferation assay showed that 
the EVs did not have any significant effect on the number of viable cells (data not shown). For the spheroid-forming assay, SW480 and SW620 cells were pre-incubated with normoxic and hypoxic EVs for $24 \mathrm{~h}$ and then plated at low density in methylcellulose-containing serum-free defined medium. Under these conditions, cells formed floating 3D multicellular spheroids originating from a single cell not by aggregation of cells (Figure 3A). This assay showed that the pre-incubation of SW480 cells with hypoxic EVs from either SW480 or SW620 cells significantly increased the number of spheroidforming cells as compared to normoxic EVs or PBS control (Figure 3B upper panel). In contrast, hypoxic SW480-derived EVs only marginally increased the spheroid forming ability of SW620 cells, whereas SW620-derived EVs did not have an impact on the spheroid forming ability of SW620 cells (Figure 3B lower panel). However, SW620 cells per se had a higher spheroid-forming ability than SW480 cells.

Effects of EVs on motility and invasiveness of CRC cells. To interrogate the effects of normoxic and hypoxic EVs on the motility and invasiveness of hypoxia-naïve SW480 and SW620 cells, scratch wound healing and BME invasion assays were performed. The scratch wound healing assay showed that SW480 cells filled the scratch significantly faster in the presence of hypoxic SW480 and SW620 EVs as compared to normoxic EVs or PBS control (Figure 4A, B left panel). As the proliferation assay did not reveal any effect of the EVs on the growth rate, this effect was likely due to increased motility, not proliferation of cells. At the same time, these EVs did not have a significant effect on the motility of SW620 cells (Figure 4B, right panel). In line with these findings, the BME cell invasion assay showed that hypoxic SW480 and SW620-derived EVs significantly increased the invasion of SW480 cells through the BME- coated membranes, while again they did not have any significant effect on the invasiveness of SW620 cells (Figure 4C).

\section{Discussion}

Hypoxic microenvironment is an important driving force of tumor progression. Increasing evidence shows that hypoxiainduced signaling promotes cancer cell invasion, metastasis, angiogenesis, self-renewal capacity, chemoresistance and metabolic reprogramming $(2,5)$. However, while the intracellular signaling events have been studied for several decades, our understanding about the intercellular signaling under hypoxic conditions has just started to shape up and EVs appear to serve as one of the main tools for communication among cancer cells and between cancer and host cells. EVs secreted by hypoxic cancer cells have previously been shown to promote the invasiveness, migration and spheroid-forming ability of hypoxia-naïve cancer cells in prostate cancer and oral squamous cell carcinoma $(23,24)$, and to confer more aggressive and chemoresistant phenotype to ovarian cancer cells (25). Moreover, hypoxic tumor-derived EVs have been shown to promote angiogenesis $(16,26)$ and induce M2 macrophage polarization (27).

Here, hypoxia stimulated the release of EVs from CRC cells and this effect was mediated by HIF1. Given that hypoxia has been shown to enhance the release of EVs by breast (28) and ovarian (25) cancer cells as well as by cardiomyocytes (29) and mesenchymal stem cells (MSC) (30), the release of EVs appears to be a common hypoxia response, but its biological role is likely to be different in various situations. For example, in case of myocardial ischemia, MSC and cardiac progenitor cell-derived EVs have been shown to have cardioprotective and regenerative effects $(30,31)$, and therefore, may have a therapeutic potential (32), whereas hypoxic cancer-derived EVs appear to have mainly tumor-promoting effects.

Our data showed that hypoxic EVs are taken-up by hypoxia-naïve CRC cells more efficiently than normoxic EVs. This suggests that hypoxic conditions alter the composition of EV surface molecules in a way that facilitates their uptake. Indeed, our recent proteomic analysis of the surface proteins of EVs released by hypoxic and normoxic of SW480 and SW620 cells revealed 16 hypoxia-induced proteins including integrin ITGA6, LAMP1, COPB1, ADAM10 etc. (submitted for publication by Nakurte et al.). However, whether the enhanced uptake is selective only for cancer cells remains to be investigated.

Hypoxic SW480 and SW620 cell-derived EVs stimulated the motility and invasiveness of hypoxia-naïve primary tumor-derived SW480 cells, whereas they had a little effect on the metastasis-derived SW620 cells. Similarly, they increased the spheroid-forming capacity of SW480, but not of SW620 cells. Formation of clonal multicellular spheroids is associated with increased expression of cancer stem cell markers, chemoresistance and tumorigenicity and therefore spheroid-forming assay has been widely used as a marker for cancer stemness (33-35). Our data showed that primary tumor-derived CRC cells had a greater capacity to internalize and respond to hypoxic EVs than metastasis-derived cells, thus suggesting that the biological function of hypoxic cancer-derived EVs could be to propagate an aggressive and invasive phenotype. However, at this point, it is not clear to what extent these findings can be generalized, therefore more isogenic cell line pairs should be tested to elucidate if this is a common feature of cancers in general.

Furthermore, it is not clear whether these effects were caused by internalization of EVs and transfer of signaling molecules into the recipient cells or via binding of EVs to the cell surface receptors. In prostate cancer, a number of proteins that are present at higher levels in hypoxic than normoxic EVs including proteins associated with the epithelial adherens junction and cytoskeleton signaling have 
been identified (24). In glioma, hypoxic EVs were found to be enriched with hypoxia-regulated mRNAs and proteins such as MMPs, IL-8, caveolin 1 (26), as well protein-lysine 6-oxidase, thrombospondin-1 and VEGF (36). However, direct proof that these proteins are the cause of functional changes in the recipient cells is yet lacking. Hypoxic EVs are also known to deliver miRNAs to recipient cells (7). In fact, hypoxic oral squamous cell carcinoma-derived EVs have been shown to contain high levels of miR-21 and its depletion in the cells resulted in reduced migration and invasion of the recipient hypoxic oral squamous carcinoma cells, suggesting that these effects indeed might be mediated by transfer of miR-21 (23). Alternatively, it is possible that the intracellular signaling is triggered by binding of EVs to the cell surface receptors, not by internalization of EVs, as it has recently been shown in $\mathrm{T}$ cells $(37,38)$.

In summary, hypoxia enhanced the release of EVs from CRC cells in a HIF1-dependent manner. Hypoxic EVs were taken-up by hypoxia-naïve CRC cells more efficiently than normoxic EVs and they enhance motility, invasiveness and stemness of primary tumor-derived SW480 cells. Our data support the hypothesis that hypoxia promotes the progression of CRC via paracrine EV-mediated signaling conferring aggressiveness and invasiveness to hypoxia-naïve cancer cells in the primary tumor, whereas metastatic cells are less responsive to these signals.

\section{Acknowledgements}

This work was supported in part by the Latvian Council of Science (grant No.625) and the Latvian National Research Programme BIOMEDICINE 2014-2017, and by Academy of Finland programme grant no. 287089 (PS, MP) and COST ACTION BM1202.

\section{References}

1 Vaupel P and Mayer A: Hypoxia in cancer: significance and impact on clinical outcome. Cancer Metastasis Rev 26(2): 225239, 2007.

2 Philip B, Ito K, Moreno-Sanchez $\mathrm{R}$ and Ralph SJ: HIF expression and the role of hypoxic microenvironments within primary tumours as protective sites driving cancer stem cell renewal and metastatic progression. Carcinogenesis 34(8): 16991707, 2013.

3 Sullivan R, Pare GC, Frederiksen LJ, Semenza GL and Graham $\mathrm{CH}$ : Hypoxia-induced resistance to anticancer drugs is associated with decreased senescence and requires hypoxia-inducible factor1 activity. Mol Cancer Ther 7(7): 1961-1973, 2008.

4 Nagaraju GP, Bramhachari PV, Raghu G and El-Rayes BF: Hypoxia inducible factor-1alpha: Its role in colorectal carcinogenesis and metastasis. Cancer Lett 366(1): 11-18, 2015.

5 Qiu GZ, Jin MZ, Dai JX, Sun W, Feng JH and Jin WL: Reprogramming of the tumor in the hypoxic niche: the emerging concept and associated therapeutic strategies. Trends Pharmacol Sci 38(8): 669-686, 2017.
6 Choudhry $\mathrm{H}$ and Harris AL: Advances in hypoxia-inducible factor biology. Cell Metab 27(2): 281-298, 2018.

7 Yanez-Mo M, Siljander PR, Andreu Z, Zavec AB, Borras FE, Buzas EI, Buzas K, Casal E, Cappello F, Carvalho J, Colas E, Cordeiro-da Silva A, Fais S, Falcon-Perez JM, Ghobrial IM, Giebel B, Gimona M, Graner M, Gursel I, Gursel M, Heegaard NH, Hendrix A, Kierulf P, Kokubun K, Kosanovic M, Kralj-Iglic V, Kramer-Albers EM, Laitinen S, Lasser C, Lener T, Ligeti E, Line A, Lipps G, Llorente A, Lotvall J, Mancek-Keber M, Marcilla A, Mittelbrunn M, Nazarenko I, Nolte-'t Hoen EN, Nyman TA, O'Driscoll L, Olivan M, Oliveira C, Pallinger E, Del Portillo HA, Reventos J, Rigau M, Rohde E, Sammar M, Sanchez-Madrid F, Santarem N, Schallmoser K, Ostenfeld MS, Stoorvogel W, Stukelj R, Van der Grein SG, Vasconcelos MH, Wauben MH and De Wever O: Biological properties of extracellular vesicles and their physiological functions. J Extracell Vesicles 4: 27066, 2015.

8 Kalra H, Simpson RJ, Ji H, Aikawa E, Altevogt P, Askenase P, Bond VC, Borras FE, Breakefield X, Budnik V, Buzas E, Camussi G, Clayton A, Cocucci E, Falcon-Perez JM, Gabrielsson S, Gho YS, Gupta D, Harsha HC, Hendrix A, Hill AF, Inal JM, Jenster G, Kramer-Albers EM, Lim SK, Llorente A, Lotvall J, Marcilla A, Mincheva-Nilsson L, Nazarenko I, Nieuwland R, Nolte-'t Hoen EN, Pandey A, Patel T, Piper MG, Pluchino S, Prasad TS, Rajendran L, Raposo G, Record M, Reid GE, Sanchez-Madrid F, Schiffelers RM, Siljander P, Stensballe A, Stoorvogel W, Taylor D, Thery C, Valadi H, van Balkom BW, Vazquez J, Vidal M, Wauben MH, Yanez-Mo M, Zoeller M and Mathivanan S: Vesiclepedia: a compendium for extracellular vesicles with continuous community annotation. PLoSBiol 10(12): e1001450, 2012.

9 Sadovska L, Eglitis $\mathrm{J}$ and Line A: Extracellular vesicles as biomarkers and therapeutic targets in breast cancer. Anticancer Res 35(12): 6379-6390, 2015.

10 Maia J, Caja S, Strano Moraes MC, Couto N and Costa-Silva B: Exosome-based cell-cell communication in the tumor microenvironment. Front Cell Dev Biol 6: 18, 2018.

11 Dos Anjos Pultz B, Andres Cordero da Luz F, Socorro Faria S, Peixoto Ferreira de Souza L, Cristina Brigido Tavares P, Alonso Goulart V, Fontes W, Ricardo Goulart L and Jose Barbosa Silva M: The multifaceted role of extracellular vesicles in metastasis: Priming the soil for seeding. Int J Cancer 140(11): 2397-2407, 2017.

12 Peinado H, Zhang H, Matei IR, Costa-Silva B, Hoshino A, Rodrigues G, Psaila B, Kaplan RN, Bromberg JF, Kang Y, Bissell MJ, Cox TR, Giaccia AJ, Erler JT, Hiratsuka S, Ghajar CM and Lyden D: Pre-metastatic niches: organ-specific homes for metastases. Nat Rev Cancer 17(5): 302-317, 2017.

13 Lucchetti D, Calapa F, Palmieri V, Fanali C, Carbone F, Papa A, De Maria R, De Spirito M and Sgambato A: Differentiation affects the release of exosomes from colon cancer cells and their ability to modulate the behavior of recipient cells. Am J Pathol 187(7): 1633-1647, 2017.

14 Chiba M, Watanabe N, Watanabe M, Sakamoto M, Sato A, Fujisaki M, Kubota S, Monzen S, Maruyama A, Nanashima N, Kashiwakura I and Nakamura T: Exosomes derived from SW480 colorectal cancer cells promote cell migration in HepG2 hepatocellular cancer cells via the mitogen-activated protein kinase pathway. Int J Oncol 48(1): 305-312, 2016.

15 Zhang S, Zhang Y, Qu J, Che X, Fan Y, Hou K, Guo T, Deng G, Song N, Li C, Wan X, Qu X and Liu Y: Exosomes promote cetuximab resistance via the PTEN/Akt pathway in colon cancer cells. Braz J Med Biol Res 51(1): e6472, 2017. 
16 Huang $\mathrm{Z}$ and Feng Y: Exosomes derived from hypoxic colorectal cancer cells promote angiogenesis through Wnt4-induced betacatenin signaling in endothelial cells. Oncol Res 25(5): 651-661, 2017.

17 Lugini L, Valtieri M, Federici C, Cecchetti S, Meschini S, Condello M, Signore M and Fais S: Exosomes from human colorectal cancer induce a tumor-like behavior in colonic mesenchymal stromal cells. Oncotarget 7(31): 50086-50098, 2016.

18 Yamada N, Kuranaga Y, Kumazaki M, Shinohara H, Taniguchi $\mathrm{K}$ and Akao Y: Colorectal cancer cell-derived extracellular vesicles induce phenotypic alteration of $\mathrm{T}$ cells into tumorgrowth supporting cells with transforming growth factor-beta1mediated suppression. Oncotarget 7(19): 27033-27043, 2016.

19 Leibovitz A, Stinson JC, McCombs WB, 3rd, McCoy CE, Mazur KC and Mabry ND: Classification of human colorectal adenocarcinoma cell lines. Cancer Res 36(12): 4562-4569, 1976

20 Endzelins E, Berger A, Melne V, Bajo-Santos C, Sobolevska K, Abols A, Rodriguez M, Santare D, Rudnickiha A, Lietuvietis V, Llorente A and Line A: Detection of circulating miRNAs: comparative analysis of extracellular vesicle-incorporated miRNAs and cell-free miRNAs in whole plasma of prostate cancer patients. BMC Cancer 17(1): 730, 2017.

21 McDonald PC, Winum JY, Supuran CT and Dedhar S: Recent developments in targeting carbonic anhydrase IX for cancer therapeutics. Oncotarget 3(1): 84-97, 2012.

22 Viziteu E, Grandmougin C, Goldschmidt H, Seckinger A, Hose D, Klein B and Moreaux J: Chetomin, targeting HIF1alpha/p300 complex, exhibits antitumour activity in multiple myeloma. Br J Cancer 114(5): 519-523, 2016.

23 Li L, Li C, Wang S, Wang Z, Jiang J, Wang W, Li X, Chen J, Liu K, Li C and Zhu G: Exosomes derived from hypoxic oral squamous cell carcinoma cells deliver miR-21 to normoxic cells to elicit a prometastatic phenotype. Cancer Res 76(7): 17701780, 2016.

24 Ramteke A, Ting H, Agarwal C, Mateen S, Somasagra R, Hussain A, Graner M, Frederick B, Agarwal R and Deep G: Exosomes secreted under hypoxia enhance invasiveness and stemness of prostate cancer cells by targeting adherens junction molecules. Mol Carcinog 54(7): 554-565, 2015.

25 Dorayappan KDP, Wanner R, Wallbillich JJ, Saini U, Zingarelli R, Suarez AA, Cohn DE, and Selvendiran K: Hypoxia-induced exosomes contribute to a more aggressive and chemoresistant ovarian cancer phenotype: a novel mechanism linking STAT3/Rab proteins. Oncogene 37(28): 3806-3821, 2018.

26 Kucharzewska P, Christianson HC, Welch JE, Svensson KJ, Fredlund E, Ringner M, Morgelin M, Bourseau-Guilmain E, Bengzon $\mathrm{J}$ and Belting $\mathrm{M}$ : Exosomes reflect the hypoxic status of glioma cells and mediate hypoxia-dependent activation of vascular cells during tumor development. Proc Natl Acad Sci USA 110(18): 7312-7317, 2013

27 Wang X, Luo G, Zhang K, Cao J, Huang C, Jiang T, Liu B, Su $\mathrm{L}$ and Qiu Z: Hypoxic tumor-derived exosomal miR-301a mediates M2 macrophage polarization via PTEN/PI3Kgamma to promote pancreatic cancer metastasis. Cancer Res, 2018. doi: 10.1158/0008-5472.CAN-17-3841. [Epub ahead of print]
28 King HW, Michael MZ and Gleadle JM: Hypoxic enhancement of exosome release by breast cancer cells. BMC Cancer 12: 421, 2012.

29 Chistiakov DA, Orekhov AN and Bobryshev YV: Cardiac extracellular vesicles in normal and infarcted heart. Int J Mol Sci 17(1), 2016.

30 Bian S, Zhang L, Duan L, Wang $\mathrm{X}$, Min $\mathrm{Y}$ and $\mathrm{Yu} \mathrm{H}$ : Extracellular vesicles derived from human bone marrow mesenchymal stem cells promote angiogenesis in a rat myocardial infarction model. J Mol Med (Berl) 92(4): 387-397, 2014.

31 Giricz Z, Varga ZV, Baranyai T, Sipos P, Paloczi K, Kittel A, Buzas EI and Ferdinandy P: Cardioprotection by remote ischemic preconditioning of the rat heart is mediated by extracellular vesicles. J Mol Cell Cardiol 68: 75-78, 2014.

32 Belting $M$ and Christianson HC: Role of exosomes and microvesicles in hypoxia-associated tumour development and cardiovascular disease. J Intern Med 278(3): 251-263, 2015.

33 Zhang J, Espinoza LA, Kinders RJ, Lawrence SM, Pfister TD, Zhou M, Veenstra TD, Thorgeirsson SS and Jessup JM: NANOG modulates stemness in human colorectal cancer. Oncogene 32(37): 4397-4405, 2013.

34 Stankevicius V, Kunigenas L, Stankunas E, Kuodyte K, Strainiene E, Cicenas J, Samalavicius NE and Suziedelis K: The expression of cancer stem cell markers in human colorectal carcinoma cells in a microenvironment dependent manner. Biochem Biophys Res Commun 484(4): 726-733, 2017.

35 Piulats JM, Kondo J, Endo H, Ono H, Hagihara T, Okuyama H, Nishizawa Y, Tomita Y, Ohue M, Okita K, Oyama H, Bono H, Masuko T and Inoue M: Promotion of malignant phenotype after disruption of the three-dimensional structure of cultured spheroids from colorectal cancer. Oncotarget 9(22): 1596815983, 2018.

36 Kore RA, Edmondson JL, Jenkins SV, Jamshidi-Parsian A, Dings RPM, Reyna NS and Griffin RJ: Hypoxia-derived exosomes induce putative altered pathways in biosynthesis and ion regulatory channels in glioblastoma cells. Biochem Biophys Rep 14: 104-113, 2018.

37 Muller L, Mitsuhashi M, Simms P, Gooding WE and Whiteside TL: Tumor-derived exosomes regulate expression of immune function-related genes in human $\mathrm{T}$ cell subsets. Sci Rep 6: 20254, 2016.

38 Muller L, Simms P, Hong CS, Nishimura MI, Jackson EK, Watkins SC and Whiteside TL: Human tumor-derived exosomes (TEX) regulate Treg functions via cell surface signaling rather than uptake mechanisms. Oncoimmunology 6(8): e1261243, 2017.
Received July 30, 2018

Revised August 24, 2018

Accepted August 28, 2018 